\title{
Multipolar effects and strong coupling in hybrid plasmonic metamaterials
}

\author{
Arash Farhang ${ }^{1}$, Anantha Ramakrishna ${ }^{2}$, Olivier J.F. Martin ${ }^{1}$ \\ ${ }^{1}$ Nanophotonics and Metrology Laboratory, Swiss Federal Institute of Technology Lausanne \\ (EPFL), 1015 Lausanne, Switzerland; \\ ${ }^{2}$ Metamaterials and Plasmonics Laboratory, Department of Physics, \\ Indian Institute of Technology Kanpur, 208016 Kanpur, India
}

\begin{abstract}
Recently stacked metamaterial structures coupled to a conductive plane have been investigated and have been shown to exhibit the same properties as stacked structures with double the layers, due to dipole mirror coupling. Here we study a system of stacked subwavelength metallic grating layers coupled to a metal film and show that this system not only supports the localized modes of a doubly layered structure, but also, for non-normal incidence, supports modes that exhibit a clear propagation and in one case a simultaneous localization of the electromagnetic field in the region between the metal film and the first grating layer. Furthermore we show that this hybridized propagating mode, excited for any $\mathrm{N}$ number of periodic layers, is further influenced as it couples with the highest energy localized mode of the periodic layered stack. Additionally it is found that the localized modes of the structure can be spectrally positioned in a directly adjacent manner, resulting in wideband absorption that can effectively be tuned by varying the grating film spacing.
\end{abstract}

Keywords: Localized, delocalized, surface plasmon, plasmonic, multilayer, grating, film, propagating, hybrid, hybridization, absorption.

\section{INTRODUCTION}

Plasmonics, the bridge between the best of optics and electronics, is based on resonant electron plasma oscillations in metallic nanostructures ${ }^{1,2}$. There are two types of plasmon resonances, localized plasmons and propagating plasmons ${ }^{3}$. The former, confined around nanoparticles, nanoantennas, and various other compact nanostructures, allows for very high near-field enhancements at optical frequencies due to high localizations of the scattered field and has been shown useful in applications ranging from trapping and sensing ${ }^{4-6}$ to cancer treatment ${ }^{7,8}$. The latter, based on the free propagation of surface waves at an extended metal dielectric interface, has been key to the development of surface plasmon based biosensors ${ }^{9-12}$ and shows great promise in the development of ultra-fast and compact optical circuitry ${ }^{13-17}$. Metamaterials composed of subwavelength structures are of great interest since they can be tuned to exhibit normally unattainable electromagnetic properties. At optical frequencies, artificial structures such as periodic metallic gratings, nanodots, and splitring resonators have been investigated. A combination of localized plasmonic structures, especially $2 \mathrm{D}$ arrays, stacked to give a 3D geometry ${ }^{18,19}$, has allowed for the progressive development of optical metamaterials ${ }^{20-30}$, which come in as key components for the development of many new plasmonic devices.

Stacked composites of such structures have also been proposed and investigated for additional tunability of spectral properties. Branching out even farther we have systems of such stacked structures above a conductive plane ${ }^{31}$. Although these have not been well investigated thus far, studies till this point have shown that a conductive plane in very close proximity to a multilayer acts as a mirror and thus doubles the number of layers ${ }^{31}$. In the case of optical metamaterials, the metallic nanostructures support localized plasmon resonances while a continuous conductive plane such as a metal film supports propagating plasmon resonances. Here we take the simplest optical metamaterial structure, an array of subwavelength $\mathrm{Au}$ gratings, and explore its response when up to four layers are stacked directly above a Au film. As shown previously in the case of two cut-wire layers ${ }^{31}$, use of the plasmon hybridization model $^{32,33}$ suggests the existence of $2 \mathrm{n}$ number of dipolar modes, which we will refer to as dipole-dipole modes, for $\mathrm{n}$ number of layers above a conductive plane. If we apply the rules of mirror coupling, however, only n number of dipole-dipole modes can exist. In addition to verifying this claim and explaining some minor digressions, we also examine as a function of both angle of incidence and wavelength, the effects of near-field coupling between multiple dipole-dipole plasmon modes and

Photonic and Phononic Properties of Engineered Nanostructures II,

edited by Ali Adibi, Shawn-Yu Lin, Axel Scherer, Proc. of SPIE Vol. 8269 ,

82691B · C 2012 SPIE · CCC code: 0277-786X/12/\$18 - doi: 10.1117/12.908923

Proc. of SPIE Vol. 8269 82691B-1 
propagating SPP modes. Furthermore we demonstrate how this coupling can be used to tune the wideband absorption that we observe in this system.

The outline of this paper is as follows. In sections 2 we briefly introduce the geometry and method used; in section 3 we discuss modes primarily propagative in nature; in section 4 modes primarily localized in nature; in section 5 the emergence and tuning of wideband absorption; and finally conclude in section 6.

\section{STRUCTURAL GEOMETRY AND SIMULATION METHOD}

The geometry being studied consists of $\mathrm{n}$ number of Au grating layers of $200 \mathrm{~nm}$ periodicity. The gratings are $\mathrm{l}=100 \mathrm{~nm}$ long, $\mathrm{w}=15 \mathrm{~nm}$ wide and infinite along the $\mathrm{y}$-axis. The gap between each adjacent layer is $\mathrm{g}=30 \mathrm{~nm}$. They are placed $\mathrm{s}=20 \mathrm{~nm}$ from the surface of a $\mathrm{t}=25 \mathrm{~nm}$ thick Au film all embedded in $\mathrm{SiO}_{2}$ background of $\varepsilon=2.13$. The optical dielectric constants of $\mathrm{Au}$ are taken from the dielectric data of Johnson and Christy ${ }^{34}$. The simulation method used in this paper is based on the Surface Integral Equation (SIE) technique, extended for use in periodic structures ${ }^{35}$. The structure is excited via p-polarized planewave illumination, as shown in Fig. 1, over a range $\lambda=400-1200 \mathrm{~nm}$ and angles $\theta=0-85^{\circ}$ with respect to the normal.

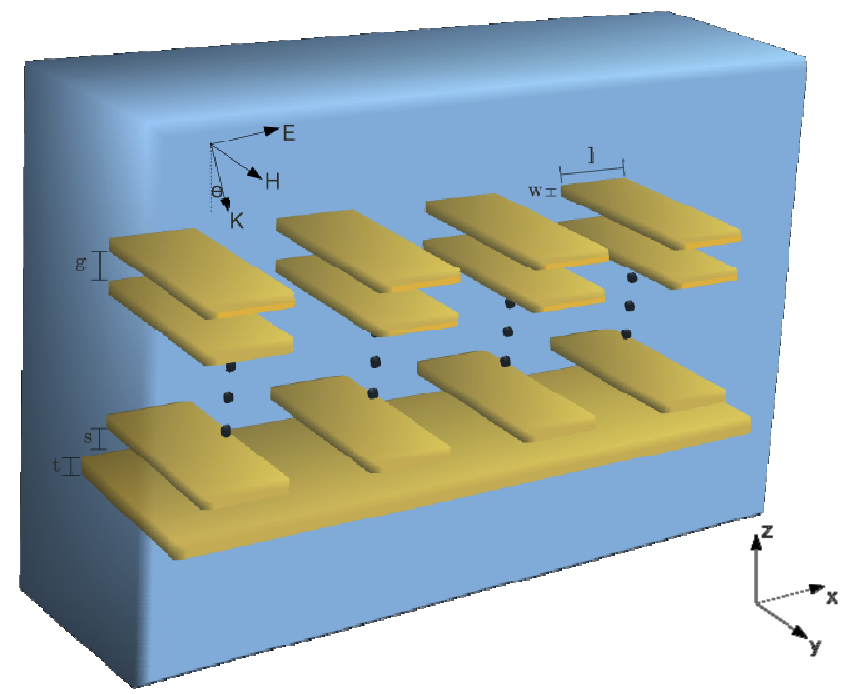

Figure 1 . The geometry being studied, a mutlilayer stack, composed of $\mathrm{Au}$, periodic in the $\mathrm{x}$ direction, infinite along the $\mathrm{y}$-axis, and with structural parameters $\mathrm{s}=20 \mathrm{~nm}, \mathrm{t}=25 \mathrm{~nm}, \mathrm{l}=100 \mathrm{~nm}, \mathrm{w}=15 \mathrm{~nm}, \mathrm{~g}=30 \mathrm{~nm}$, a $200 \mathrm{~nm}$ periodicity, and a glass background with a permittivity of $\varepsilon=2.13$.

\section{PROPAGATING MODES}

In any such system with both continuous and discrete metallic structures, we expect two kinds of modes, the first being the propagating surface plasmon (SPP) modes of the continuous structure and the second being localized surface plasmon (LSP) modes supported by the discrete structures ${ }^{15,36-38}$. Due to the fact that we have a film thickness of just $\mathrm{t}=25 \mathrm{~nm}$ thickness, the SPP modes on the two sides of the film couple, resulting in two SPP modes, an asymmetric (odd) and a symmetric (even) mode ${ }^{39}$. Since we are illuminating in an entirely $\mathrm{SiO}_{2}$ environment the launching of SPP waves can only be achieved either through Bragg scattering or possibly through hybridization with the Au grating layers. We plot in Fig. 2(a-d) the absorption spectra of this hybrid system for $n=1,2,3,4$ grating layers respectively. Apart from the parasitic absorbance, caused by the interband transitions of $A u$ primarily below $\lambda=500 \mathrm{~nm}$, we are able to distinguish in the range $\lambda=500-700 \mathrm{~nm}$ two distinct modes that redshift with increasing angle of incidence. Via additional simulations (not shown here) where we replace the Au gratings with dielectric gratings of a very high permittivity, $\varepsilon=30$, we find that we are able to excite the same two modes, implying that that they must be due to Bragg scattering. Further calculations (not shown here) of the SPP dispersions, assuming a periodicity of $200 \mathrm{~nm}$, show us that these modes are indeed the 
Bragg scattered SPP modes folded back into the first Brillouin zone. Due to the presence of the Au grating layers, however, we see a constant change in the dispersion of the SPP modes as the number of grating layers is increased.

Additionally, the lower wavelength/long range SPP mode, normally plagued by losses due to its close vicinity to the $500 \mathrm{~nm}$ mark, becomes much more pronounced as the number of grating layers is increased, due to the increased level of Bragg scattering. We verify the nature of these modes further by plotting the electric field profile, in vector form, within an $\mathrm{x}-\mathrm{z}$ cross section for an $\mathrm{n}=4$ grating layer structure at wavelengths of $\lambda=570 \mathrm{~nm}, \lambda=670 \mathrm{~nm}$ and $\theta=55^{\circ}$ incidence (Figs. $3(a, b))$. As can be seen, we obtain a symmetric field profile for the lower energy/higher wavelength even mode and an asymmetric field profile for the higher energy/lower wavelength odd mode. Furthermore, it can be seen that in the case of the even mode, a capacitance effect is present due to the coupling between the first grating layer and the film, thereby resulting in a local concentration of the optical field in the region between the two. Although the discussion of the remaining peaks in the absorption spectra of Fig. 2 is to be left for section 4, it should be mentioned here that these are the dipole-dipole modes of the multilayer structure and are primarily localized in nature. The presence of these modes, predominantly the one of highest energy, excited in the case of multiple grating layers only for angles of incidence of $\theta=70^{\circ}$ and above, leads to a very pronounced repulsion/blueshift of the even SPP mode at high angles of incidence and hence results in the high level of distortion in the SPP dispersion observed for an increased number of grating layers.

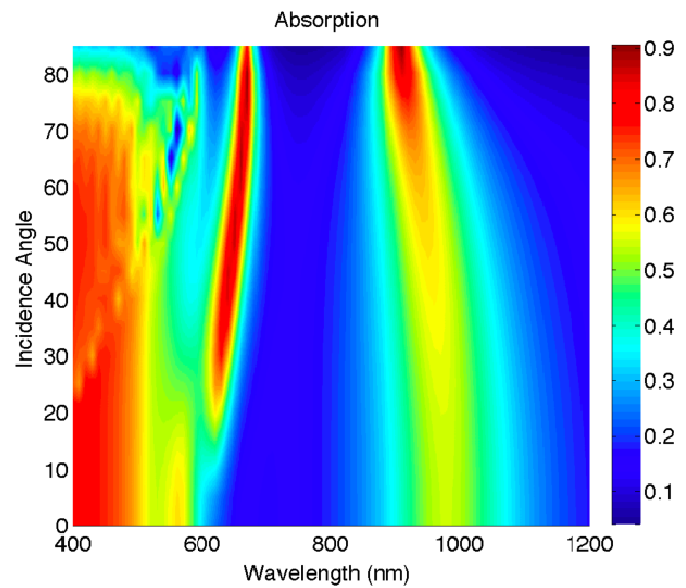

(a)

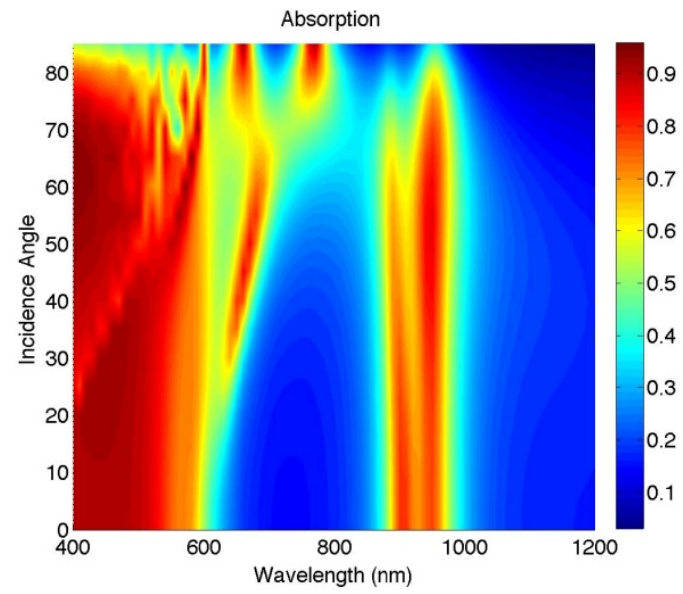

(c)

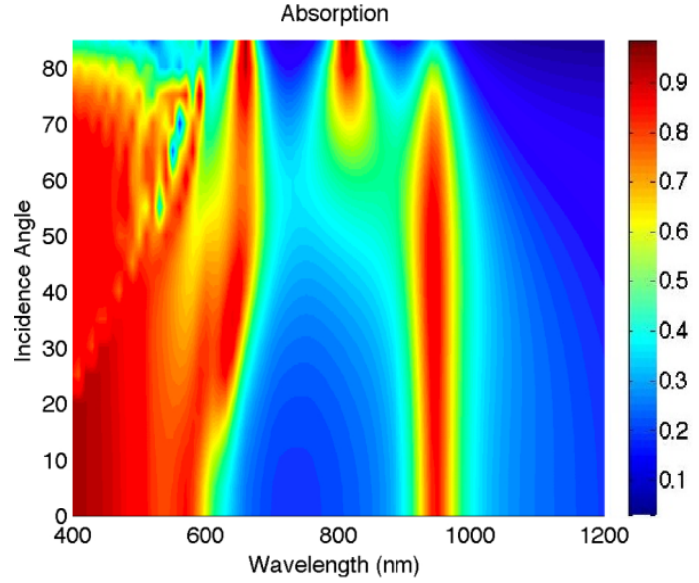

(b)

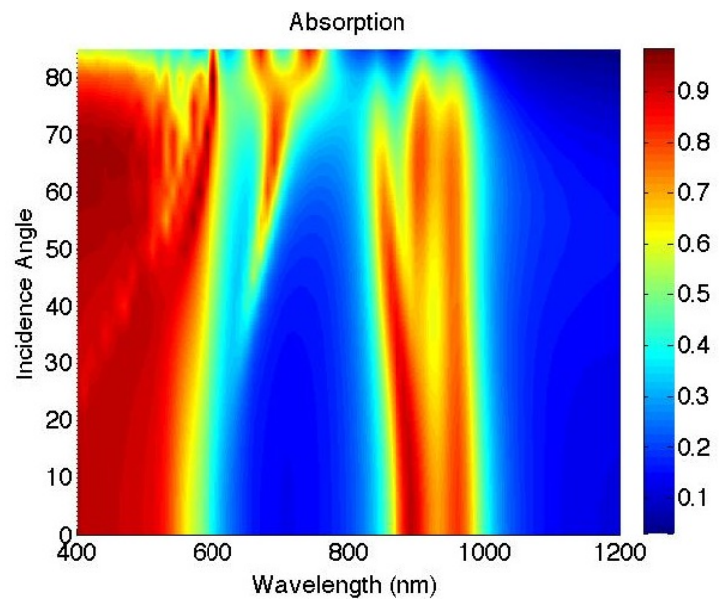

(d)

Figure 2(a-d). Absorption spectra for structures with $\mathrm{n}=1,2,3,4$ number of Au grating layers respectively, spaced $\mathrm{s}=20 \mathrm{~nm}$ above a Au film. As can be seen, there are two main absorption bands between $\lambda=500 \mathrm{~nm}$ and $\lambda=700 \mathrm{~nm}$ that correspond to the odd and even SPP modes of the film respectively. The additional modes are the LSP dipoledipole modes of the Au grating layers, which hybridize due their close vicinity to the Au film. 


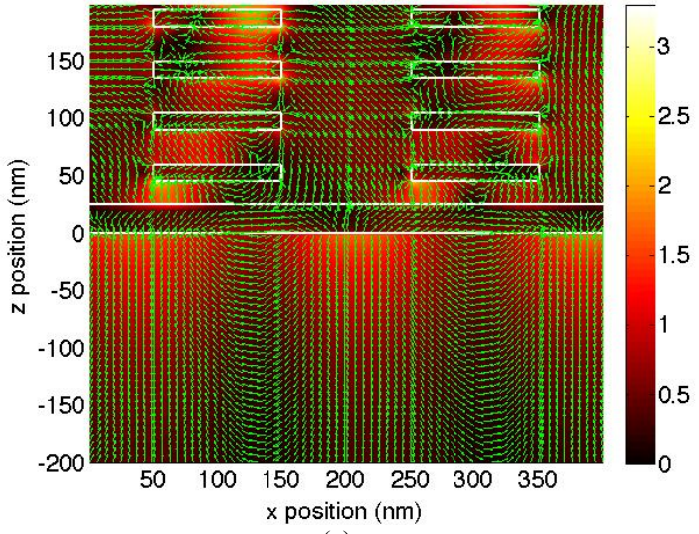

(a)

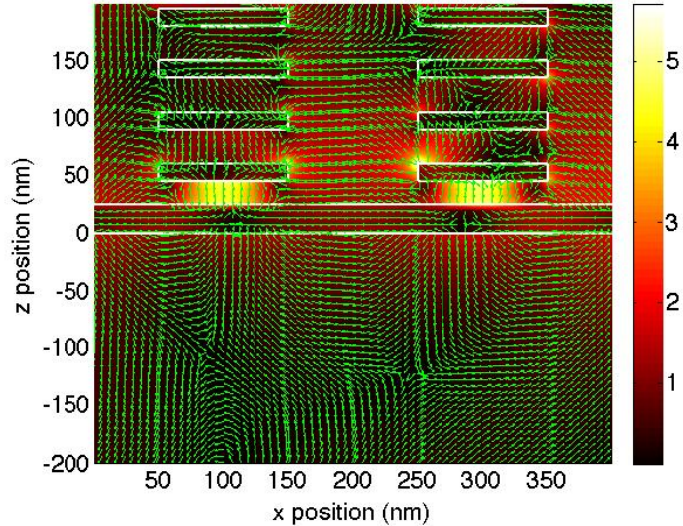

(b)

Figure 3(a,b). Cross sections of the four layer Au grating and film structure showing the electric field profile. The arrows indicate the orientation of the electric field, while the color scale indicates the magnitude of the field. (a) Odd SPP mode for a $\theta=55^{\circ}$ and $\lambda=570 \mathrm{~nm}$. (b) Even SPP mode for $\theta=55^{\circ}$ and $\lambda=670 \mathrm{~nm}$.

\section{LOCALIZED MODES}

In Section 3 we investigated the two absorption bands lying in the range $\lambda=500-700 \mathrm{~nm}$ and established that they correspond to the odd and even propagating SPP modes. Recalling that we expect both SPP and LSP modes for any such structure similar to the one shown in Fig. 1, it is only logical that the remaining modes must be primarily localized in nature. Indeed in agreement with the plasmon hybridization model and the effects of mirror coupling ${ }^{31}$, previously discussed in section 1 , we see that we obtain $n$ number of these additional modes for $n$ number of grating layers. The theory suggests that these LSP modes consist of dipolar charge distributions in the metallic gratings that couple to their mirror image in the metal film, hence the term dipole-dipole modes. As a result, we expect the following modes for $\mathrm{n}=1,2,3,4$ grating layers (Fig. $4(\mathrm{a}-\mathrm{d})$ ).

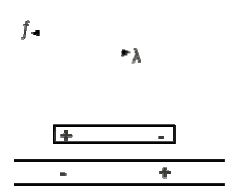

(a)

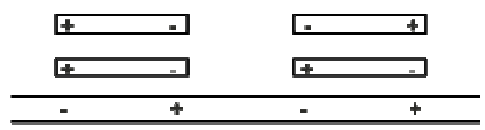

(b)

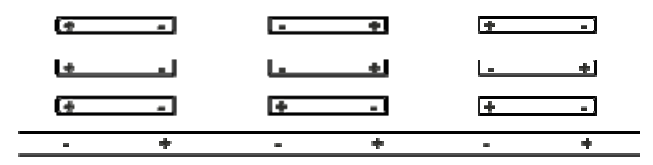

(c)

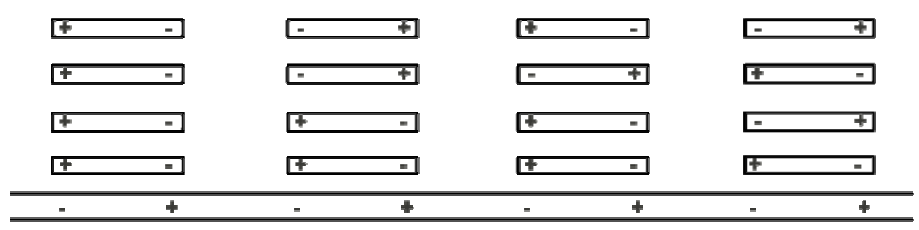

(d)

Figure 4(a-d). LSP dipole-dipole modes predicted for the system under study, predicted by the plasmon hybridization and mirror coupling model for $\mathrm{n}=1,2,3,4$ number of grating layers respectively. Modes are plotted from left to right in order of increasing wavelength/decreasing frequency. 
The electric field profiles (not shown here) of these modes within cross sections just like those shown in Fig. 3(a,b), indicate a very good agreement with the hybridization and mirror coupling model, in almost every case, the exception being for the case of the highest energy/lowest wavelength dipole-dipole mode for multilayer structures with $n=3,4$ grating layers. Rather than having the charge distributions shown on the far left of Figs. 4(c,d) respectively, it turns out that these two modes have instead the charge distributions shown in Fig. 5(a,b). This is simply due to the fact that these modes are only excited for angles of incidence $\theta>70^{\circ}$. The high incidence angle limits the overall quantity of excitation light that can penetrate down into the grating layers. As a consequence of this limitation, only the first few grating layers are excited by the incident light, and thus mirror coupling is induced in the center of the stack, i.e. between two adjacent grating layers, rather than at the surface of the Au film. It should be noted that unlike the lower energy dipole-dipole modes of the multilayer structure, the highest energy dipole-dipole is excited only for high angles of incidence due to the fact that the incident phase front impinging on the stacked grating layers must essentially be void of phase retardations in order to excite the same dipolar charge orientation in multiple neighboring grating layers.

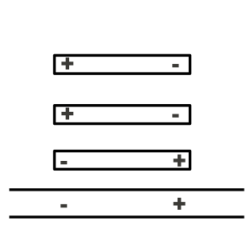

(a)

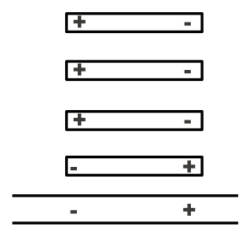

(b)

Figure 5(a,b). Numerically obtained charge distribution of the lowest wavelength/highest energy LSP dipole-dipole modes excited for $\mathrm{n}=3,4$ grating layers.

\section{WIDEBAND ABSORPTION}

As seen in Fig. 2 and explained in Sec. 4, the presence of n number of Au grating layers above a Au film results in the existence of $n$ number of LSP dipole-dipole modes. For a grating film spacing of $s=20 \mathrm{~nm}$ and $n \geq 3$ number of grating layers, the resonance position of the $n-1$ lowest energy modes lie spectrally adjacent to one another, thereby resulting in a relatively wideband of absorption. For $\mathrm{n}=4$ number of grating layers, the close proximity of these modes, combined with their relatively broad linewidths gives an almost dispersionless absorption band of $120 \mathrm{~nm}$ width, with an absorption value of up to $90 \%$. This structure, therefore, could be a very good candidate for a broadband plasmonic absorber.

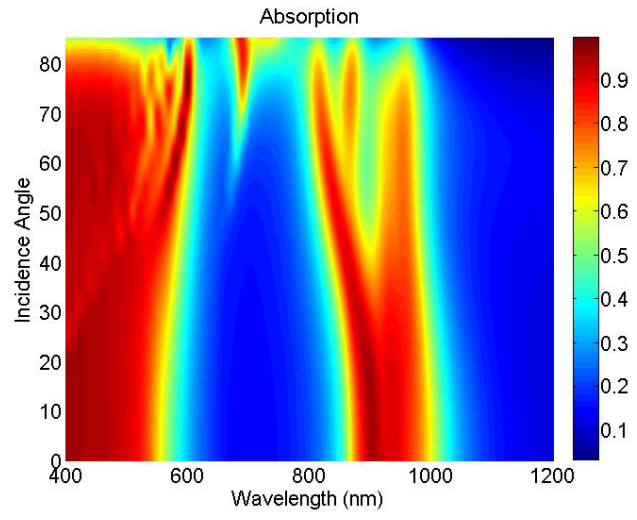

(a)

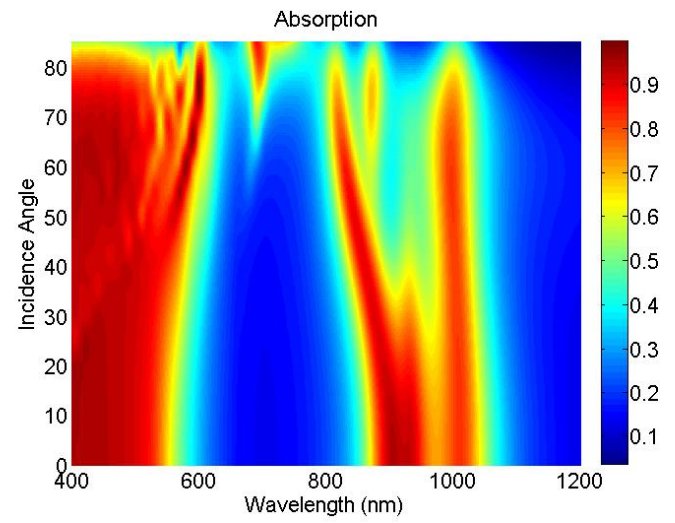

(b)

Figure 6(a,b). Absorption spectra for structures with $\mathrm{n}=5$ number of Au grating layers spaced $\mathrm{s}=20 \mathrm{~nm}$ and $\mathrm{s}=15 \mathrm{~nm}$ above a Au film respectively. (a) As can be seen the addition of just another grating layer results in a much more pronounced and flatter absorption band than for the case of $\mathrm{n}=4$ grating layers. (b) The bandwidth of absorption can be controlled further by varying the spacing between the grating layers and Au film. Here we see the case for s=15nm a bandwidth of $160 \mathrm{~nm}$. 
In order to demonstrate the tuning of this wideband absorption, we investigate the case where we have $n=5$ number of grating layers. We show in Fig. $6(a, b)$ the absorption spectra assuming $n=5$ number of grating layers and spacings $\mathrm{s}=20 \mathrm{~nm}$ and $\mathrm{s}=15 \mathrm{~nm}$ respectively. Already with the addition of just a single grating layer, we are able to achieve a much more uniform and higher absorbing band than for the case of $n=4$ number of grating layers, assuming angles of incidence $\theta<40^{\circ}$. As in the case of $\mathrm{n}=3,4$ grating layers, for angles $\theta>40^{\circ}$, the bands begin to disperse and gradually become visibly distinct. If we now reduce the grating film spacing down to $\mathrm{s}=15 \mathrm{~nm}$, we effectively increase the level of coupling between the LSP and SPP modes and thus induce a redshift, more so to the lower energy SPP modes than the higher energy modes, thereby effectively broadening the absorption bandwidth from $120 \mathrm{~nm}$ to $160 \mathrm{~nm}$.

\section{CONCLUSION}

We have demonstrated numerically a hybrid plasmonic structure composed of multiple layers of sub-wavelength Au gratings coupled to a Au film. The hybrid structure supports for any n number of grating layers both even and odd SPP modes as well as n number of dipolar LSP modes. The dispersion of the even SPP mode is found to be influenced greatly through coupling with the highest energy LSP mode, while the odd SPP mode remains unaffected. Additionally it is found that the plasmon hybridization and mirror coupling model accurately describe the existence and charge distribution of the LSP dipole-dipole modes up to a certain degree. It is for $n \geq 3$ number of grating layers that these models fail to accurately describe the charge distributions associated with the highest energy LSP mode. An interpretation is provided to explain these deviations. Finally, we observe that this multilayer structure is able to provide a relatively high level of wideband absorption and, additionally, how this absorption band can be tuned via coupling between the grating layers and the Au film.

\section{ACHNOLEDGMENT}

This research was carried out as part of a collaborative project between the plasmonic research group at EPFL and the plasmonics research group at IIT Kanpur. Funding from the State Secretariat for Education and Research SER within the Indo Swiss Joint Research Programme is gratefully acknowledged.

\section{REFERENCES}

[1] H. A. Atwater, "The promise of plasmonics," SIGDA Newsl., 37(9), 1-1 (2007).

[2] D. K. Gramotnev, and S. I. Bozhevolnyi, "Plasmonics beyond the diffraction limit," Nature Photonics, 4(2), 8391.

[3] A. Farhang, and O. J. F. Martin, "Plasmon delocalization onset in finite sized nanostructures," Optics Express, 19(12), 11387-11396 (2011).

[4] D. E. Chang, J. D. Thompson, H. Park et al., "Trapping and Manipulation of Isolated Atoms Using Nanoscale Plasmonic Structures,” Physical Review Letters, 103(12), 123004 (2009).

[5] L. Huang, S. Maerkl, and O. J. F. Martin, "Integration of plasmonic trapping in a microfluidic environment," Optics Express, 17(8), 6018-6024 (2009).

[6] M. L. Juan, R. M., and R. Quidant, "Plasmon nano-optical tweezers," Nature Photonics, 5(349), 349-356 (2011).

[7] C. Loo, Alex Lin, Leon Hirsch et al., "Nanoshell-Enabled Photonics-Based Imaging and Therapy of Cancer," Technology in Cancer Research \& Treatment, 3(1), (2004).

[8] D. P. O'Neal, L. R. Hirsch, N. J. Halas et al., "Photo-thermal tumor ablation in mice using near infraredabsorbing nanoparticles," Cancer Letters, 209(2), 171-176 (2004).

[9] H. J. Chen, X. S. Kou, Z. Yang et al., "Shape- and size-dependent refractive index sensitivity of gold nanoparticles," Langmuir, 24(10), 5233-5237 (2008).

[10] W. Chungang, and J. Irudayaraj, "Gold nanorod probes for the detection of multiple pathogens," Small, 4(12), 2204-8 (2008).

[11] W. P. Hall, S. N. Ngatia, and R. P. Van Duyne, "LSPR Biosensor Signal Enhancement Using Nanoparticle-Antibody Conjugates," The Journal of Physical Chemistry C, 115(5), 1410-1414 (2011).

[12] J. Homola, [Surface plasmon resonance based sensors.] Springer, (2006). 
[13] S. I. Bozhevolnyi, and J. Jung, "Scaling for gap plasmon based waveguides," Opt. Express, 16(4), (2008).

[14] S. I. Bozhevolnyi, V. S. Volkov, E. Devaux et al., "Channel plasmon subwavelength waveguide components including interferometers and ring resonators," Nature, 440(7083), 508-511 (2006).

[15] G. Lévêque, and R. Quidant, "Channeling light along a chain of near-field coupled gold nanoparticles near a metallic film," Opt. Express, 16(26), 22029-22038 (2008).

[16] F. Liu, R. Y. Wan, Y. D. Huang et al., "Refractive index dependence of the coupling characteristics between long-range surface-plasmon-polariton and dielectric waveguide modes," Optics Letters, 34(17), 2697-2699 (2009).

[17] T. Nikolajsen, K. Leosson, and S. I. Bozhevolnyi, "Surface plasmon polariton based modulators and switches operating at telecom wavelengths," Appl. Phys. Lett., 85, 5833 (2004).

[18] N. Liu, H. Guo, L. Fu et al., "Three-dimensional photonic metamaterials at optical frequencies," Nat Mater, 7(1), 31-37 (2008).

[19] N. Liu, M. Hentschel, T. Weiss et al., "Three-Dimensional Plasmon Rulers," Science, 332(6036), 1407-1410 (2011).

[20] A. Alù, N. Engheta, and "Plasmonic materials in transparency and cloaking problems: mechanism, robustness, and physical insights," Optics Express, 15(6), 3318-3332 (2007).

[21] M. Beruete, M. Navarro-Cia, M. Sorolla et al., "Negative refraction through an extraordinary transmission lefthanded metamaterial slab," Physical Review B, 79(19), (2009).

[22] C. G. Biris, and N. C. Panoiu, "Second harmonic generation in metamaterials based on homogeneous centrosymmetric nanowires," Physical Review B, 81(19), 195102.

[23] A. Christ, O. J. F. Martin, Y. Ekinci et al., "Symmetry Breaking in a Plasmonic Metamaterial at Optical Wavelength," Nano Lett., (2008).

[24] B. Edwards, A. Alu, M. G. Silveirinha et al., "Experimental Verification of Plasmonic Cloaking at Microwave Frequencies with Metamaterials," Physical Review Letters, 103(15), 153901-4 (2009).

[25] M. J. Freire, R. Marques, and L. Jelinek, "Experimental demonstration of a $m u=-1$ metamaterial lens for magnetic resonance imaging," Applied Physics Letters, 93(23), (2008).

[26] B. Gallinet, and O. J. F. Martin, "Ab initio theory of Fano resonances in plasmonic nanostructures and metamaterials," Physical Review B, 83(23), 235427 (2011).

[27] A. Greenleaf, and Y. Kurylev, "Electromagnetic Wormholes and Virtual Magnetic Monopoles from Metamaterials," Phys. Rev. Lett., 99, 183901 (2007).

[28] J. Hao, "Super-reflection and cloaking based on zero index metamaterial," Applied Physics Letters, 96(10), 3 (2010).

[29] S. A. Ramakrishna, S. Guenneau, S. Enoch et al., "Confining light with negative refraction in checkerboard metamaterials and photonic crystals," Phys. Rev. A, 75, 063830-063835 (2007).

[30] S. Zhang, D. A. Genov, Y. Wang et al., "Plasmon-induced transparency in metamaterials," Physical Review Letters, 101(4), (2008).

[31] N. Liu, H. Guo, L. Fu et al., "Plasmon Hybridization in Stacked Cut-Wire Metamaterials," Advanced Materials, 19(21), 3628-3632 (2007).

[32] A. Maaroof, J. Nygaard, and D. Sutherland, "Plasmon Hybridization in Silver Nanoislands as Semishells Arrays Coupled to a Thin Metallic Film," Plasmonics, 6(2), 419-425 (2011).

[33] P. Nordlander, and E. Prodan, "Plasmon Hybridization in Nanoparticles near Metallic Surfaces," Nano Letters, 4(11), 2209-2213 (2004).

[34] P. B. Johnson, and R. W. Christy, "Optical Constants of the Noble Metals," Phys. Rev. B, 6(12), 4370-4379 (1972).

[35] B. Gallinet, A. M. Kern, and O. J. F. Martin, "Accurate and versatile modeling of electromagnetic scattering on periodic nanostructures with a surface integral approach,” J. Opt. Soc. Am. A, 27(10), 2261-2271 (2010).

[36] A. Christ, G. Lévêque, O. J. F. Martin et al., "Near-field-induced tunability of surface plasmon polaritons in composite metallic nanostructures," Journal of Microscopy, 229(2), 344-353 (2008).

[37] G. Lévêque, and O. J. F. Martin, "Tunable composite nanoparticle for plasmonics," Opt. Lett., 31(18), 2750 2752 (2006).

[38] G. Lévêque, and O. J. F. Martin, "Optical interactions in a plasmonic particle coupled to a metallic film," Optics Express, 14(21), 9971-9981 (2006).

[39] H. Raether, "Dispersion relation of surface plasmons on gold- and silver gratings," Optics Communications, 42(4), 217-222 (1982). 